\title{
Stick-slip Actuation of Electrostatic Stepper Micropositioners for Data Storage - the $\mu$ Walker
}

\author{
Mihai Patrascu and Stefano Stramigioli, Senior Member, IEEE \\ Univ. of Twente, The Netherlands \\ Control Engineering Laboratory \\ $\{$ M.Patrascu, S.Stramigioli\}@utwente.nl
}

\begin{abstract}
This paper is about the $\mu W$ alker, an electrostatic stepper motor mainly intended for positioning the data probes with respect to the storage medium in a data storage device. It can deliver forces up to $1.7 \mathrm{mN}$ for ranges as large as $140 \mu \mathrm{m}$. Controlling the stick-slip effects at the sliding surfaces is of central importance for reliable operation. A model is introduced to estimate the operating voltage of the actuator plate, which is an essential part of the $\mu W$ alker. Several methods to obtain displacements smaller than one nominal step $(\approx 50$ $n \mathrm{~m})$ are discussed, as well as how to increase the step repeatability and accuracy.
\end{abstract}

\section{Introduction}

As the number of applications where MEMS technology is implemented increases, it has become clear that scaling down to MEMS is not always possible, nor desired. Instead, novel design (often from scratch) is performed, which leads to totally new concepts, challenges and opportunities. One of the industries which could profit very much from MEMS devices is data recording and storage. Several institutes and companies ${ }^{1}$ presently make efforts in order to present a novel storage medium prototype, which does not suffer from the disadvantages of the current hard-disk technology, but yet can be scaled down further, possibly down to atomic data storage, where each atom or even electron spin represents one bit of data [8], [3], [1] and [2]. Our project, called $\mu S P A M^{2}$, has as target to design a prototype data storage device with data densities over 1 Tbit/inch ${ }^{2}$, com-

\footnotetext{
${ }^{1}$ amongst them: IBM, University of Twente, Carnegie Mellon University, and INSIC - The Information Storage Industry Consortium

${ }^{2} \mu S P A M$ stands for Micro Scanning Probe Array Memory; please visit http: / / www. uspam.nl for more details
}

pared to about $200 \mathrm{Gbit} /$ inch $^{2}$ of the current state-ofthe-art hard disk technology. Due to the design layout chosen, many thousands of read/write probes can read and write in parallel. The reduced power consumption of this device could serve in mobile applications, allowing tens of gigabytes of data to be addressed into one small chip, whereas the access time is below that of the hard-disk.

In data storage and many other fields, the positioner speed and accuracy are key factors for the overall system performance. In $\mu S P A M$, this positioner is called the $\mu W$ alker, also known as the shuffle motor $[5,6]$ and has been designed while keeping in mind these important factors. As the 2D $\mu$ Walker [6] has not yet been sufficiently characterized, only the $1 \mathrm{D}$ case will be treated here [7].

After explaining the actuation method, a model of the electro-elastic propulsion of the mid plate is presented, which predicts the minimum actuation voltage needed to produce basic steps. Then, a new type of actuation sequence leading to improved step reproducibility will be shown. Finally, several actuation methods for achieving intra-step size displacement, i.e. steps smaller than one basic step, will be presented.

\section{Actuation principles}

Fig. 1 shows that the actuator is suspended by four beam-like suspension springs (the tiny vertical lines attached to the corners), such that when no actuation is applied, the device automatically jumps back to rest, where the four suspension springs are straight, like in Fig. 1. Moreover, these springs electrically connect the bond pads (not in the picture) to the $\mu$ Walker subparts. In the shape presented here, the device can deliver forces higher than $1 \mathrm{mN}$ and the walking range currently achieved is over $20 \mu \mathrm{m}$, which makes it suitable for probe recording applications. 


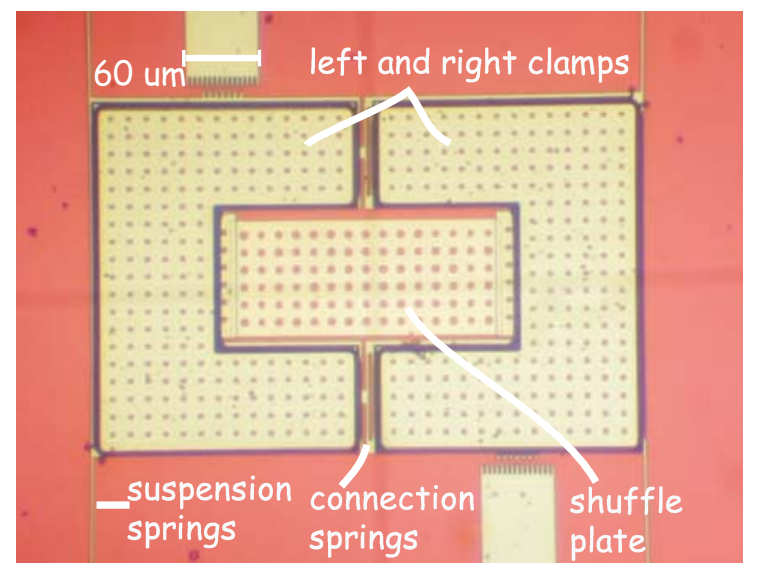

Figure 1. $\mu$ Walker magnification of about 130x. Courtesy of E. Sarajlic, University of Twente.

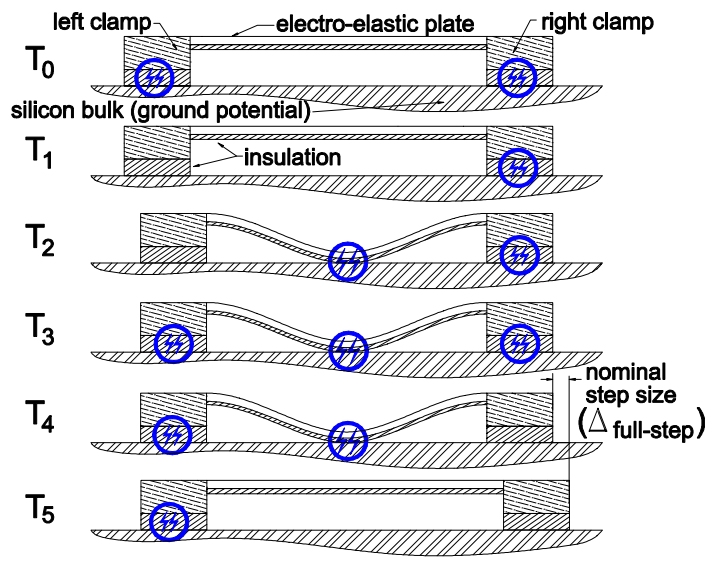

Figure 2. Schematic representation of one step to the right.
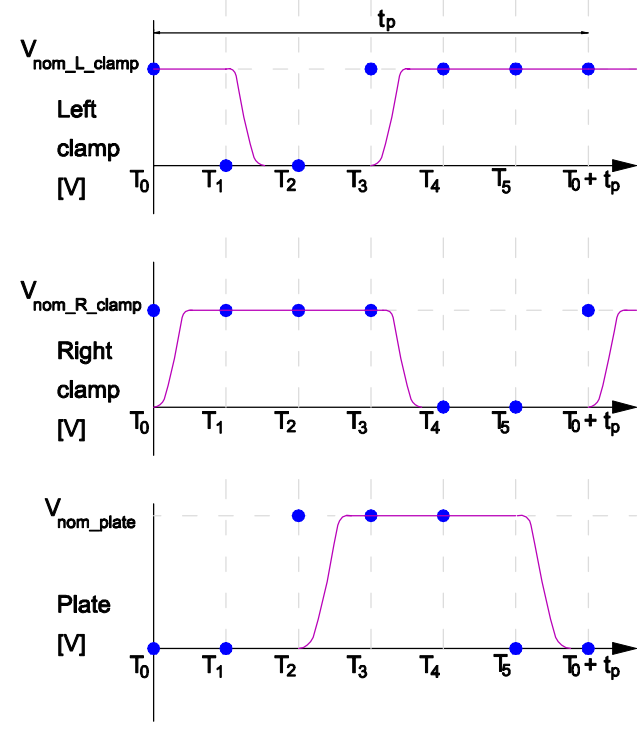

Figure 3. Basic, unipolar actuation sequence for one step.

The actuation of this electrostatic device is based on six sub-sequences, as can be seen in Fig.2. There are three actuation voltages, two for the clamps and one for the shuffle plate in the middle. The walking surface is an insulating silicon nitride layer, with underneath a highly doped silicon bulk, which has ground potential during the measurements. The insulating layer prevents short circuit between the actuation voltages and the ground potential. Moreover, the silicon nitride layer has the advantage that it has a relatively high hardness, so that wear is reduced and experiment repeatability is greatly improved. In this way, also the reliability and lifetime of the motor have drastically increased. About $34.10^{9}$ steps were taken with the same device without performance deterioration [7].

\subsection{Basic walking sequence}

In the sequel, the basic walking principle shall be discussed. Fig. 3 shows the sequence generation for one step as in Fig. 2. The dots are the digital output signals supplied to Labview from a file, whereas the continuous shapes are the actual voltages offered to the device by the amplifier, which is connected to the Matlab software. With the current setup, up to 80.000 steps per second have been obtained, with input voltage slew rates around $60 \mathrm{~V} / \mu \mathrm{s}[6]$. 


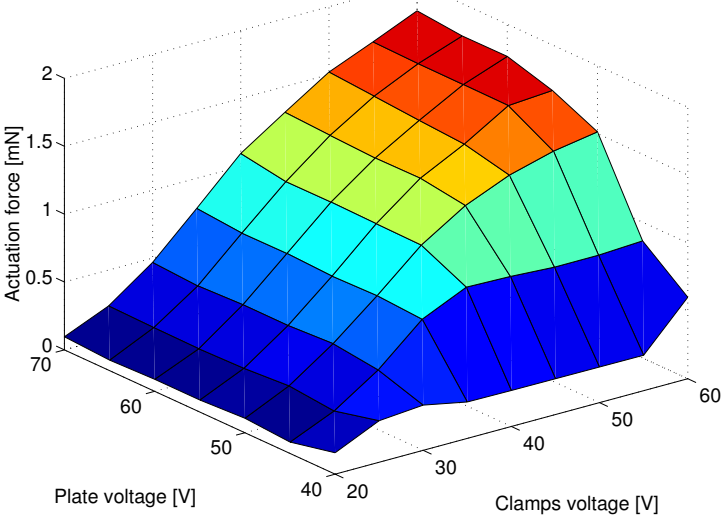

Figure 4. The maximum actuator force as a function of clamps and plate voltages.

In Fig. 3, during each subsequence exactly one of the three voltages changes. In the figure, subsequences $\left(T_{0} \ldots T_{5}\right)$ form one nominal step, after which the subsequences are repeated for the next step. Starting with $T_{0}$, a clamp voltage is applied to both clamps, $V_{\text {nom_clamp }}=40 \mathrm{~V}$. Due to the electrostatic force between every clamp and the ground, the clamps are attracted and stick to the ground. Moving either of the clamps in the horizontal direction is not possible now. During $T_{1}$, the left clamp is released, followed by $T_{2}$, where a voltage is applied to the shuffle plate.

When on, the shuffle plate voltage is always greater than the pull-in voltage of the plate. The pull-in voltage shall be described in next subsection and is about $38 \mathrm{~V}$ for the $\mu W$ alker dimensions under study, while we apply a shuffle plate voltage $V_{\text {nom_plate }}=55 \mathrm{~V}$.

The consequence of applying a plate voltage while at least one clamp is free, is that the plate bends and about half of its length sticks to the surface, whereas the ends which connect the shuffle to the clamps are being bent according to an ' $S$ '-shape and retain some bending energy, which will be used later in sequence $T_{5}$. The total effect is, that the distance between the clamps has decreased one step size. Now, the left clamp voltage is also actuated $\left(T_{3}\right)$, followed by releasing the right clamp $\left(T_{4}\right)$ and then the shuffle plate $\left(T_{5}\right)$. At this stage, the $\mu W$ alker has performed one step to the right.

The size of one step is defined as $\Delta_{\text {full-step }}$ in the sequel. Thus, completing one sequence as described above leads to a shift of $\Delta_{\text {full-step }}$ to the right. Applying the same sequence in reversed order gives a step to the left. When the device is at the starting position, $\Delta_{\text {full-step }} \approx 50 \mathrm{~nm}$, depending on several dimensions and on the actuation voltages.
Obviously, applying different input voltages to the clamps and plate yield different step sizes and output forces. First, an increased plate voltage increases step size, as show the measurements in [4]. Second, increasing the voltage applied to the clamps is favourable for the stick force which keeps the clamps fixed to the walking surface when actuated. The relation between the clamps and plate voltages, and the maximum output force can be seen in Fig. 4. Here, the device moves 200 consecutive steps in one direction and in this way the suspension springs are loaded. The forces deduced from the total deflection of the springs are an indication of the total force that can be exerted by the $\mu$ Walker for a certain set of input voltages. It can be seen that the maximum force delivered appears starting with plate and clamp voltages around $60 \mathrm{~V}$ and $55 \mathrm{~V}$, respectively.

\subsection{Electro-elastic propulsion}

The target of this subsection is to derive the pullin voltage of the $\mu W$ alker plate while in clamped-free mode, such that for a variety of dimension sets, a proper actuation voltage can be found. Conversely, given a setup with certain amplifier characteristics, a dimension set can then be found such that the pull-in phenomenon takes place.

Following the lines of [4] but disregarding an extra insulation layer on the bulk, we start by defining the elastic and electrostatic energy of the plates:

with $K$ as defined below and $0<\xi<2$ is a configuration variable for taking into account the stick region of the beam; for $\xi<1$, the plate does not touch the surface;

$$
E_{\text {bending }}=\frac{1}{2} K \xi^{2}, \quad E_{\text {elec }}=\frac{1}{2} C V^{2} \frac{1}{\sqrt{1-\xi}}
$$

$K$ and $C$ are the mechanical bending stiffness and the electrical capacity, respectively:

$$
K=\frac{64}{15} \frac{E_{S i} w b^{3} d^{2}}{L^{3}}, \quad C=\frac{L w \epsilon_{0} \epsilon_{a i r}}{d} .
$$

with $E_{S i}$ the Si modulus of elasticity, $w$ the width, $b$ the thickness and $L$ the length of the bending plate; $d$ is the distance -at rest- between plate and wafer bulk and $\epsilon_{0}, \epsilon_{\text {air }}$ are the permittivity of vacuum and the relative permittivity of the gas or liquid between the plate and surface, respectively.

Now, the pull-in voltage $V_{\text {pull-in }}$ can be derived by setting the difference between the partial derivatives of the two energies with respect to $\xi$ equal to zero:

$\frac{\partial E_{\text {bending }}-E_{\text {elec }}}{\partial \xi}=0 \Longleftrightarrow V=\sqrt{\frac{4 K \xi(1-\xi)^{\frac{3}{2}}}{C}}$ 
Table 1. Example of device dimensions and constants.

\begin{tabular}{|l|l||l|l|}
\hline Dimensions & Value & Constants & Value \\
\hline \hline$b[\mu \mathrm{m}]$ & 2.0 & $E_{S i}[P a]$ & $150 * 10^{9}$ \\
$d[\mu \mathrm{m}]$ & 2.0 & $\epsilon_{0}\left[\frac{F}{m}\right]$ & $8.85 * 10^{-12}$ \\
$L[\mu \mathrm{m}]$ & 236 & $\epsilon_{\text {air }}[]$. & 1.0 \\
\hline
\end{tabular}

It can be derived that for $\xi=\frac{2}{5}, V=V_{\text {pull-in }}$. Then, we can be writte:

$V_{\text {pull-in }}=\sqrt{\frac{8 K\left(\frac{3}{5}\right)^{\frac{3}{2}}}{C}} \Leftrightarrow V_{\text {pull-in }}=\frac{16}{25} \sqrt{2 \sqrt{15} \frac{E b^{3} d^{3}}{L^{4} \epsilon_{0} \epsilon_{r}}}$

As an example, the values of Table 1 give $V_{\text {pull-in }} \approx$ $33 \mathrm{~V}$.

\subsection{Surface charge versus step reproducibility}

During measurements, it was discovered that due to repeatedly applying the walking sequence from Fig. 3, some charge is being built up on the walking surface, which leads to worse step reproducibility. The result is that although voltage actuation is no more applied and the device is several micrometers away from the position at rest and thus the springs retain bending energy, it remains stuck to the surface for several seconds; only after that, the stick force is small enough such that the suspension springs are able to pull the device from the stick phase to the original position. The stick force in the horizontal direction resulted from the induced charge in the insulation layer has been estimated to be roughly as high as $1.9 \mu \mathrm{N}$ during the first seconds. This problem has been solved by changing the actuation, to include both positive and negative voltages. Fig. 5 shows the bipolar actuation that completes two steps. After one such sequence, the insulating material between the device and the ground potential (the wafer bulk) is very lowcharged compared to the unipolar actuation presented earlier, such that unwanted insulation charge effects like the one explained above do not occur anymore.

\subsection{Intra-step size motion}

Given a certain trajectory to follow in the shortest possible time is a well-known optimization problem in data storage. Due to the nature of the tracking problem, it is desired that the actuator should also be able to perform movements which are smaller than the maximum step size $\Delta_{\text {full-step }}$. More important, the step repeatability should be maximized. There are a number of ways to (temporarily) reduce the step size, amongst them:

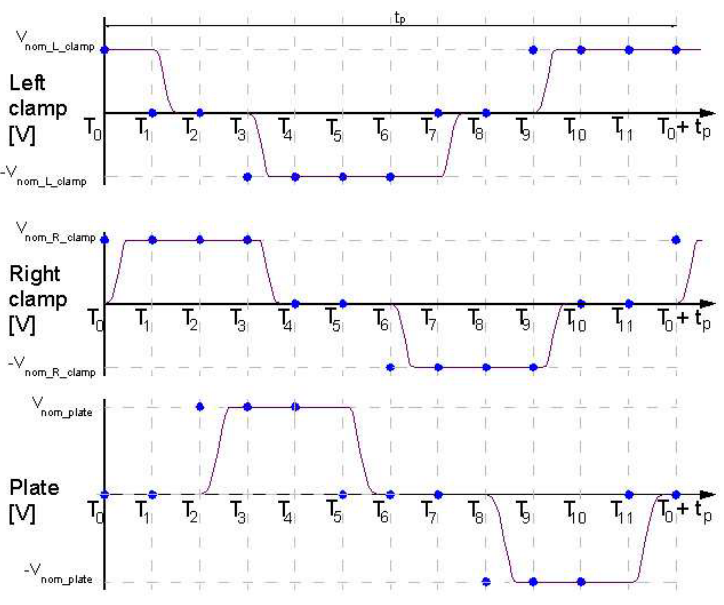

Figure 5. Bipolar actuation sequence for two steps to the right.

- introducing a small delay between the three input voltages, such that some of the six flanks from the sub-sequences in Fig. 2 partly overlap each other; for this, a very high sample frequency is needed, which increases power consumption and device complexity;

- apply actuation voltages smaller than the shuffle pull-in voltage, such that the step becomes smaller; although one would expect this to be a proper method, in practice local surface roughness, stick and charge effects greatly reduce repeatability of this method; moreover, the actuator force decreases drastically due to the lower voltage, leading to an unusable walking stroke;

- actively changing the stiffness of the suspension springs by using bimetal material properties; a rigorous change of the design would be needed as well as more input voltages for actuating the bi-metal springs;

- slightly changing the input voltage amplitudes, for example by applying a (small) voltage to the left clamp in sub-sequences $\left(T_{1}\right),\left(T_{2}\right)$ instead of keeping it zero; due to this change, the shuffle plate would have to 'pull' the left leg which also sticks, but the stick force is not so high as at the right clamp; therefore the left leg can still shift, although the step size will be decreased compared to the 'basic' actuation presented above.

Measurements show that the last option is the most effective and reproducible way to decrease the step size. 

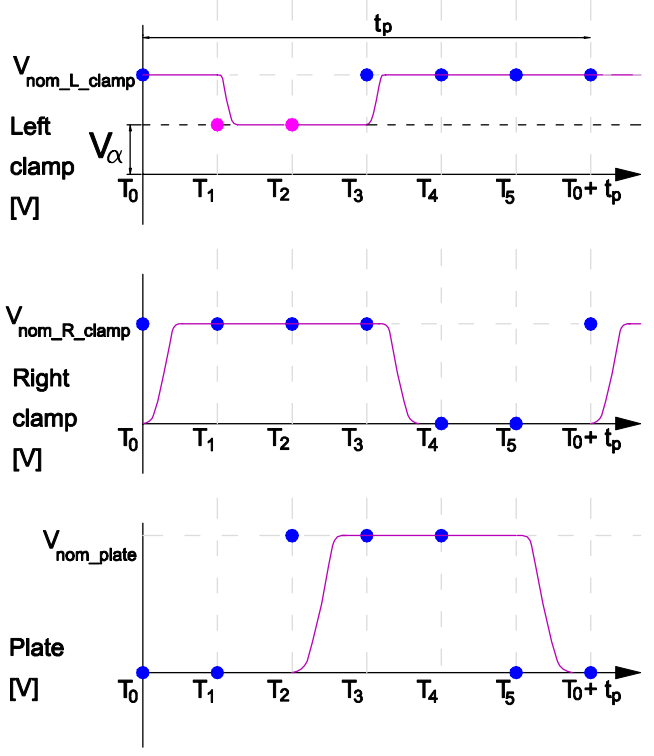

Figure 6. Sequence that performs a an intra-step.

The obvious way to solve the optimal tracking problem in the case of the $\mu W$ alker is to take as many standard steps as possible to come in the neighbourhood of the target and, if necessary, to complete the movement with an intra-step movement, a step which has a particular, smaller size than the preceding steps. In this way, we can position the moving device with an error which is equal to or less then half the size of this last step, given that the exact position is known or can be derived just before taking the step. The error is here defined as the difference between the target position and the actual position. Thus, by decreasing the minimum size of the last step, the error decreases accordingly.

\subsection{Intra-step walking sequence}

In order to make clear how the intra-steps are obtained, please see Fig. 6. Note that there are many other possibilities to achieve step reduction by altering one or more input voltages during certain sequences. For reducing complexity, the positive unipolar actuation case is explained, whereas the bipolar case can be derived in a straightforward way (see previous subsection). Compared to the basic walking cycle (Fig. 2), we can see that at $\left(T_{1}\right)$ and $\left(T_{2}\right)$, we now have a voltage $V_{\alpha}$, where $0<\left|V_{\alpha}\right|<V_{\text {nom_L_clamp. The consequence is that }}$ as the distance between the right and left leg decreases due to the plate actuation at $\left(T_{2}\right)$, due to the increased

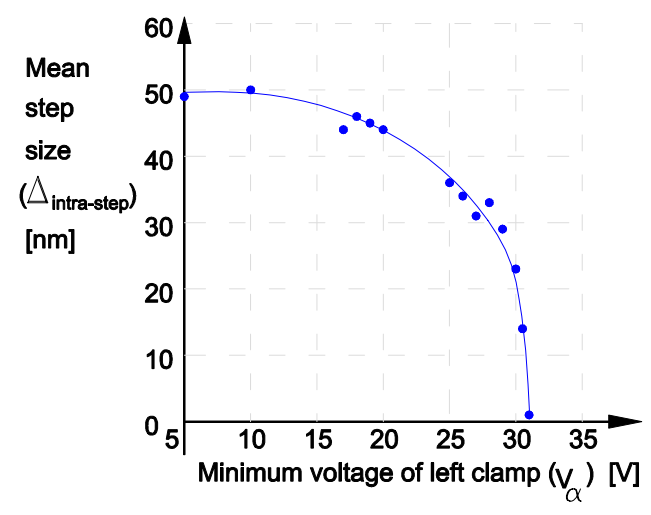

Figure 7. Step size as a function of parameter $\mathrm{V}_{\alpha}$.

left leg voltage $V_{\alpha}$ some stick will occur, decreasing the step size of the intra-step, $\Delta_{\text {intra-step }}$. Let us define $\Delta_{\text {step }}\left(V_{\alpha}\right)$ over $0 \leq V_{\alpha}<V_{\text {nom_X_clamp }}, \quad X \in$ $\{L, R\}$. For $V_{\alpha}<10 \mathrm{~V}$ and given the present set of dimensioning parameters, it can be assumed that $\Delta_{\text {full-step }} \approx \Delta_{\text {intra-step. }}$. For $V_{\alpha}>31 V, \Delta_{\text {step }} \approx$ 0nm.

Fig. 7 gives the relation between $V_{\alpha}$ and $\Delta_{\text {intra-step }}$ for $10 \mathrm{~V} \leq V_{\alpha}<31 \mathrm{~V}$. Each of the data points shown is the mean of five identical measurements, except at $28 \mathrm{~V}$, where two of the five values were outside $M E A N \pm$ $2 * S T D$ and were excluded. The five measurements needed for each of the points in Fig. 7 have been obtained after measuring a sequence of 200 consecutive steps and calculating the mean step size over all steps. Given the present set of dimensioning parameters, the step $\Delta_{\text {step }}\left(V_{\alpha}\right)$ can be defined as:

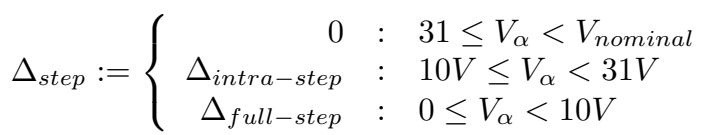

with $\Delta_{\text {intra-step }}$ a best-fit curve of deliberate shape and order, see Fig. 7.

Finally, as has been presented in [4], also the device dimensions and material properties influence the step size. This shall not be further examined here.

\section{Conclusion}

An analytical expression of the pull-in voltage as function of device parameters has been presented, valid for a generalized micro plate actuated electrostatically and which is in clamped-free mode. 
Measurements show that the actuation force of the $\mu W$ alker depends less on the plate voltage than on the voltage applied to the clamps.

A step smaller than the nominal step size can be obtained reproducibly by slightly altering the actuation sequence of a nominal step. This intra-step size is sensitive to device parameters and the applied voltages.

\section{Acknowledgment}

The authors wish to thank Edin Sarajlic for designing and fabricating the $\mu W a l k e r$, as well for the valuable input regarding the measurements. Also thanks to N. E. Ligterink for helping with model development. The $\mu W$ alker is a part of $\mu S P A M$, which is an STW project (TES5178).

\section{References}

[1] L. Abelmann, T. Bolhuis, A. M. Hoexum, G. J. M. Krijnen, and J. C. Lodder. Large capacity probe recording using storage robots. IEE Proc.-Sci. Meas. Technol, 150(5):218-221, September 2003.

[2] L. R. Carley, J. A. Bain, G. K. Fedder, D. W. Greve, D. F. Guillou, L. Abelmann, and S. Min. Single-chip computers with microelectromechanical systems-based magnetic memory (invited). Journal of Applied Physics, 87(9):6680-6685, 052000.

[3] E. Eleftheriou, P. Bächtold, G. Cherubini, A. Dholakia, C. H. T. Loeliger, and e. A. Pantazi. A nanotechnologybased approach to data storage. volume 29, pages 3-9. Morgan Kaufmann, San Francisco, August 2003.

[4] N. E. Ligterink, M. Patrascu, P. C. Breedveld, and S. Stramigioli. An energy-based electroelastic beam model for MEMS applications. September 2004. Accepted for publication.

[5] J. A. Pelesko and D. H. Bernstein. Modeling MEMS and NEMS. Chapman \& Hall/CRC, 2003. ISBN: 1-58488306-5.

[6] E. Sarajlic, E. Berenschot, H. Fujita, G. Krijnen, and M. Elwenspoek. Bidirectional electrostatic linear shuffle motor with two degrees of freedom. MEMS 2005 - 18th IEEE International Conference on Micro Electro Mechanical Systems, Miami, pages 391-394, January 2005.

[7] E. Sarajlic, E. Berenschot, N. Tas, H. Fujita, G. Krijnen, and M. Elwenspoek. High performance bidirectional electrostatic inchworm motor fabricated by trench isolation technology. TRANSDUCERS 05 - Int. Conf. on Solid State Sensors and Actuators - Seoul, Korea, July 2005. Accepted.

[8] P. Vettiger, G. Cross, M. Despont, U. Drechsler, U. Dürig, B. Gotsmann, W. Häberle, and M. A. L. e.a. The "Millipede" - nanotechnology entering data storage. IEEE Trans. Nanotechnol., 1:39-55, 2002. 